\title{
Mild hypothermia improves transplantation outcomes
}

The induction of therapeutic hypothermia in deceased organ donors significantly reduces delayed graft function in kidney transplant recipients, according to new findings. "Our research shows that slight cooling of deceased kidney donors can substantially increase transplantation success and the pool of potential donors," explains Claus Niemann. "As impressive as the potential clinical benefit, is the collaboration and research infrastructure that was developed to execute this study," adds Darren Malinoski.

Kidney transplantation is associated with notable survival benefits compared to dialysis, but the demand for suitable organs outstrips supply. "Organs are often transplanted that are not considered optimal," says Niemann. "These organs can be further damaged during the transplantation process, resulting in inferior function after transplantation. Our inspiration for this study came from the beneficial effect of mild-tomoderate cooling seen with organ systems in our laboratory and from protocols used for other clinical scenarios."

To investigate the effects of mild cooling on transplantation outcomes, the researchers randomly assigned organ donors after declaration of death according to neurologic criteria to normothermia $\left(36.5-37.5^{\circ} \mathrm{C}\right)$ or hypothermia $\left(34-35^{\circ} \mathrm{C}\right)$. Temperature control was achieved using non-invasive approaches and organ donors remained at the target temperature until they left the intensive care unit for organ recovery. "This approach is entirely non-disruptive to the work flow of health-care providers and the process of transplantation itself," say the researchers.

The trial was terminated early after an interim analysis demonstrated efficacy of

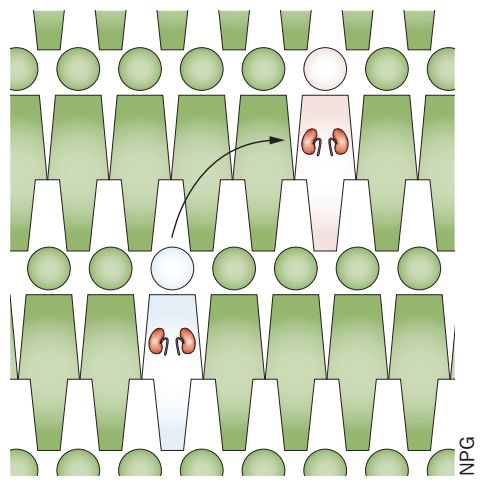

hypothermia on delayed graft function in the kidney transplant recipients, defined as the need for dialysis during the first week after transplantation. At the point of trial termination, the researchers had enrolled 370 donors and transplanted 583 kidneys. Delayed graft function occurred in $28.2 \%$ of patients who had received a transplant from a hypothermic donor compared to $39.2 \%$ who had received a transplant from a normothermic donor (adjusted OR 0.62, 95\% CI 0.43-0.92). The largest effect was seen in recipients of kidneys from extended-criteria donors, with a rate of delayed graft function of $31.0 \%$ among recipients of kidneys from hypothermic donors compared to $56.5 \%$ among recipients of kidneys from normothermic donors (adjusted OR 0.31, 95\% CI 0.15-0.68). “This approach can be literally implemented at any hospital in the world with no cost and with immediate benefits," says Niemann. "If broadly adopted, we estimate that tens of thousands of additional patients in need of kidney transplants could benefit by either receiving better functioning organs or receiving an organ that may not have been considered for transplantation."

Susan J. Allison

Original article Niemann, C. U. et al.

Therapeutic hypothermia in deceased organ donors and kidney-graft function. N. Engl. J. Med. 373, 405-414 (2015) 